\section{GENRE}

en séries

\section{Genre en séries}

Cinéma, télévision, médias

$4 \mid 2016$

Masculinités imag(in)ées 1

\title{
Des histoires d'hommes entre eux. Homosociabilité et masculinité hégémonique dans le cinéma français des années 1970
}

\section{Hélène Fiche}

\section{(2) OpenEdition}

Journals

Édition électronique

URL : https://journals.openedition.org/ges/2363

DOI : 10.4000/ges.2363

ISSN : 2437-6563

Éditeur

Presses universitaires de Bordeaux

Édition imprimée

Date de publication : 1 juin 2016

Pagination : 10-33

Référence électronique

Hélène Fiche, "Des histoires d'hommes entre eux. Homosociabilité et masculinité hégémonique dans le cinéma français des années 1970 », Genre en séries [En ligne], 4 | 2016, mis en ligne le 07 février 2022, consulté le 10 février 2022. URL : http://journals.openedition.org/ges/2363 ; DOI : https:// doi.org/10.4000/ges.2363 


\section{DES HISTOIRES D'HOMMES ENTRE EUX. HOMOSOCIABILITÉ ET MASCULINITÉ HÉGÉMONIQUE DANS LE CINÉMA FRANÇAIS DES ANNÉES 1970.}

Hélène FiCHE

\section{RÉSUMÉ}

Au cours des années 1970, dans un contexte de développement en France du mouvement féministe, de nombreux films mettent en scène des groupes d'hommes qui traversent une crise existentielle et cherchent à s'isoler des femmes pour la surmonter. Cet article propose d'étudier ce motif à travers un ensemble de sept films à succès sortis en France entre 1973 et 1977, qui ont en commun d'exalter une homosociabilité défensive articulée à des activités régressives, promouvant un modèle de masculinité normatif et hégémonique. Ces films révèlent les crispations et les inquiétudes de leurs auteurs face aux évolutions de la place des femmes dans la société française et ont contribué à légitimer l'idée d'une « crise de la masculinité » qui s'est imposée dans le cadre du backlash antiféministe du début des années 1980.

\section{MOTS CLÉS : GENRE - GINÉMA - HOMOSOGIABILITÉ - MASGULINITÉ - VIRILITÉ - BUDDY MOVIE - FÉMINISME - ANTIFÉMINISME}

\section{ABSTRAGT}

Between 1973 and 1977, as the Women's Movement expanded itself in France, French cinema began to depict groups of men going through an existential crisis and trying to isolate themselves from women in order to overcome it. This article aims to study this recurring theme through seven films, all of which praise a defensive homosociality built around regressive activities and promoting a hegemonic model of masculinity. Those movies reveal the defensive posture of their authors when it comes to the improvement of women's status and contributed to the spreading of the "masculinity crisis" rhetoric accompanying the early 1980's backlash.

\section{KEYWORDS : GENDER - CINEMA - HOMOSOGIALITY - MASGULINITY - VIRILITY - BUDDY MOVIE - FEMINISM - ANTIFEMINISM}

Hélène Fiche est ancienne élève de l'École Normale Supérieure de Lyon et agrégée d'histoire. Elle prépare une thèse de doctorat à l'Université Paris 1 Panthéon-Sorbonne, co-dirigée par Pascal Ory (Paris 1) et Geneviève Sellier (Bordeaux Montaigne) et intitulée "Construction des rapports sociaux de sexe et des normes sexuées dans les films français à succès (1969-1982)". 
Au cours des années 1970, l'émergence et le développement d'un vaste mouvement féministe en France ont contribué à une reconfiguration des normes sexuées et des rapports sociaux de sexe. Dès 1970, le champ cinématographique - et plus particulièrement les films destinés au grand public, que les producteurs cherchent à adapter à ce qu'ils pensent être les préoccupations des spectateurs et spectatrices - apparaît comme le témoin actif de ces bouleversements. En effet, en construisant un scénario et des personnages, les scénaristes et les réalisateurs, même s'ils ne veulent pas parler de leur époque, parlent toujours depuis celle-ci, et tiennent, directement ou en creux, un discours sur elle. Les films des années 1970 nous renseignent donc sur ce que leurs équipes de production jugeaient digne d'intérêt, sur les valeurs qu'elles affirmaient comme leurs et sur celles dont elles étaient inconsciemment porteuses (Ferro, 1973 : 109).

À ce titre, le renouvellement des stéréotypes sexués au sein des films de la période apparaît comme un champ d'étude particulièrement fertile. En effet, les années 1970 voient notamment émerger de nouvelles catégories de personnages masculins, associés à des motifs narratifs qui semblent traduire dans certains cas un repositionnement serein face aux évolutions récentes des rapports sociaux de sexe et, dans d'autres cas, une angoisse face à ces évolutions et une volonté de les nier. Au sein des films mettant en scène des personnages masculins inquiets et déstabilisés, un motif revient de façon récurrente et se dégage avec beaucoup de netteté en raison de son caractère sériel. On peut en effet comptabiliser, au cours des seules années 1973 à 1977, une dizaine de films ayant fait plus de 700000 entrées en salle et mettant en scène les aventures d'un duo ou d'un groupe d'hommes qui traversent une crise existentielle et cherchent à trouver refuge dans un environnement excluant les femmes. Je me concentrerai ici sur l'étude de sept de ces films, qui ne sont pas à eux seuls représentatifs du champ cinématographique de la période, mais que j’ai sélectionnés pour leur succès auprès du public, ainsi que pour la façon dont ils font de l'entre-soi masculin un moyen de résistance aux changements sociaux culturels et de reconquête d'une virilité perdue. Il s'agit de La Grande Bouffe (Marco Ferreri, 1973, 2,422 
millions d'entrées en salle) ${ }^{1}$, Les Valseuses (Bertrand Blier, 1974, 5,774 millions d'entrées)2 , Vincent, François, Paul... et les autres (Claude Sautet, 1974, 2,807 millions d'entrées) ${ }^{3}$, Calmos! (Bertrand Blier, 1976, 739000 entrées) ${ }^{4}$, Mes chers amis (Mario Monicelli, 1976, 749000 entrées)5, et enfin Un éléphant, ça trompe énormément (1976, Yves Robert, 2,925 millions d'entrées) ${ }^{6}$ et sa suite Nous irons tous au paradis (1977, Yves Robert, 2,080 millions d'entrées) 7 .

Ces films appartiennent à des genres cinématographiques et à des registres très variés, de la comédie grand public (Un éléphant, ça trompe énormément, Nous irons tous au paradis) au drame d'auteur (La Grande Bouffe), en passant par la comédie dramatique (Les Valseuses, Vincent, François, Paul... et les autres) ou la farce satirique (Calmos !). Par ailleurs, s'ils ont tous connu un succès public, certains comme Les Valseuses se sont hissés en tête du boxoffice alors que d'autres ont atteint une audience plus modeste. Il ne s'agit donc pas, en les étudiant conjointement dans cet article, de chercher à minimiser ces différences et l'impact des codes génériques auxquels ils obéissent sur leur contenu et leur réception. Au contraire, le fait qu'un même motif soit décliné à travers des univers diégétiques aussi variés offre de

${ }^{1}$ La Grande Bouffe met en scène le week-end orgiaque d'un groupe de quatre amis, qui ont choisi de se rejoindre pour manger, boire et avoir des rapports sexuels avec des prostituées, jusqu'à ce que mort s'en suive.

${ }_{2}$ Les Valseuses est un road movie, mettant en scène les aventures de deux jeunes loubards qui écument les routes de France en se livrant à divers larcins. Une jeune femme rencontrée par hasard se joint à eux et transforme peu à peu leur duo en trio.

3 Vincent, François, Paul... et les autres met en scène les aléas de la vie d'un groupe d'amis qui ont l'habitude de se retrouver à la campagne pour le week-end avec leurs épouses depuis des années. La crise économique et les problèmes conjugaux ne les épargnent pas et mettent leur amitié à rude épreuve.

${ }^{4}$ Calmos! met en scène la rencontre fortuite de deux hommes qui s'isolent à la campagne pour échapper à leurs épouses, puis aux hordes de femmes constituées en armée, qui tentent de s'emparer des hommes afin de les forcer à se livrer à des rapports sexuels. Ils se retrouvent bientôt à la tête d'un maquis d'hommes qui tentent de résister collectivement à l'offensive féminine.

${ }^{5}$ Mes chers amis met en scène cinq quadragénaires italiens qui se réunissent régulièrement le temps d'un week-end pour échapper à la vie quotidienne en se livrant à des farces régressives.

${ }^{6}$ Dans Un éléphant, ça trompe énormément, quatre amis quarantenaires restés de grands enfants se débattent face aux difficultés de la vie de couple et de famille. L'un d'entre eux est tombé amoureux d'une belle inconnue et envisage de quitter sa femme. Un autre, coureur invétéré, découvre que sa femme s'est décidée à le quitter. Un autre tente d'empêcher sa mère de s'immiscer constamment dans sa vie privée. Un dernier enfin, tente vainement de dissimuler son homosexualité à son entourage.

7 Nous irons tous au paradis est la suite d'Un éléphant, ça trompe énormément. Les quatre amis y rencontrent de nouvelles difficultés. L'un d'eux est persuadé que sa femme le trompe. Un autre s'essaye avec sa nouvelle compagne aux principes de l'amour libre. Ils décident d'acheter tous ensemble une maison à la campagne, afin de se réunir entre hommes le week-end. 
nombreuses pistes d'analyse. Mais de tels points de convergence apparaissent entre ces films sur le plan narratif qu'il semble possible de les réunir en un même ensemble et de faire l'hypothèse qu'ils traduisent tous selon des modalités variées - les crispations et les inquiétudes de leurs auteurs face aux questions de l'identité masculine et de la place des femmes dans la société française. Au moment de leur sortie en salle, ils sont d'ailleurs constamment comparés les uns aux autres par les critiques et les journalistes qui y voient à chaque fois un «hommage inconditionnel à la camaraderie $»^{8}$.

\section{Grise De LA MASGULINITÉ ET GAMARADERIE}

Des hommes en crise

Si l'on se focalise tout d'abord sur les personnages principaux de ces films, on constate qu'ils ont en commun d'être des "hommes en crise », c'est-à-dire des hommes qui traversent une période de leur vie qu'ils vivent comme « critique », sans que ce sentiment soit nécessairement provoqué par des événements hors du commun ou objectivement bouleversants. Certains de ces films comportent alors une dimension introspective, les héros exprimant leurs doutes et leurs difficultés, soit en voix off (Mes chers amis, Un éléphant, ça trompe énormément, Nous irons tous au paradis), soit directement à leurs amis (Vincent, François, Paul... et les autres, Calmos !). Dans d'autres, les personnages refusent au contraire de s'avouer leurs problèmes et tentent de les dissimuler, finissant finalement par trahir leur insécurité lorsqu'ils sont confrontés à des difficultés (Les Valseuses). Chacun de ces films comporte donc - à des degrés très variables - une dimension noire et sérieuse, y compris les comédies les plus potaches.

L'état angoissé, mélancolique et déprimé des personnages, ainsi que leur âge ${ }^{9}$, pourraient laisser penser qu'ils traversent une sévère « crise de la quarantaine » (ou de la cinquantaine), mais leur mal-être est en réalité plus profond. S'ils sont généralement incapables d'identifier sa cause, celui-ci découle toujours du constat qu'ils font de leur incompétence -

\footnotetext{
8 À propos de Mes chers amis, dans L'Express du 16 août 1976.

9 À l'exception des personnages des Valseuses qui sont de jeunes hommes, tous les autres sont des pères de famille mariés, ou des hommes en âge de l'être.
} 
professionnelle, sentimentale et/ou sexuelle - et en conséquence, de leur échec à se conformer au modèle de masculinité auquel ils aspirent. Tous souffrent en fait de n'être pas assez virils, c'est-à-dire de manquer de certains attributs associés au masculin : «la force, le courage, la capacité à se battre, le 'droit' à la violence et [les] privilèges associés à la domination de celles et ceux, qui ne sont pas, et ne peuvent pas, être virils : femmes, enfants » (Hirata, $2004: 71$ ).

Ceci est particulièrement explicite dans les films où l'état dépressif des hommes en crise s'accompagne d'une angoisse diffuse de castration. Dès le début des Valseuses par exemple, les deux héros, Pierrot (Patrick Dewaere) et Jean-Claude (Gérard Depardieu) se font attaquer à l'arme à feu par le propriétaire de la voiture qu'ils viennent de voler. Pierrot est touché aux testicules et malgré les soins qu'il reçoit, il demeure traumatisé par cette atteinte portée à ses organes génitaux et n'aura de cesse de s'interroger sur ses capacités à avoir une érection («mais si j’peux plus fourrer, plus jamais? » ou, lorsqu'il agresse sexuellement une jeune femme dans un train : «je bande pas!»). Mais même lorsqu'ils parviennent à avoir des rapports sexuels, les deux amis sont incapables - autre forme d'«impuissance sexuelle»- de procurer du plaisir aux femmes: Jeanne (Jeanne Moreau) se tue après avoir passé la nuit avec eux et Marie-Ange (Miou-Miou) reste parfaitement indifférente, malgré leurs efforts pour lui faire atteindre l'orgasme. On retrouve cette thématique de l'impuissance dans La Grande Bouffe qui raconte l'histoire de quatre amis accomplissant un suicide collectif par indigestion, au cours d'un « week-end gastronomique ». Marcello (Marcello Mastroiani), pilote de ligne, est incapable d'avoir une érection et ne peut avoir de rapports sexuels que par le biais d'objets médiateurs. Michel (Michel Piccoli), un producteur de télévision, pratique quant à lui chaque matin des exercices de gymnastique, vêtu d'un justaucorps rose, comportement jugé trop «féminin » par ses amis qui ne cachent pas leur malaise et leur inquiétude.

Dans Vincent, Paul, François... et les autres, la perte de virilité se joue avant tout dans l'échec professionnel et la castration est donc symbolique et sociale. Les quatre amis qui exercent tous une profession supposant des attributs et des qualités réputées «viriles »- la prise de risque et l'initiative 
pour Vincent le chef d'entreprise, la rigueur scientifique pour François le médecin, le génie créateur pour Paul l'écrivain, la force pour Jean le boxeur -, échouent à incarner ces valeurs (Forbes, 1992 : 184). L'entreprise de Vincent fait faillite, François a perdu toute passion pour son métier, Paul est en manque d'inspiration et ne parvient plus à écrire, et Jean manque de courage et hésite à accepter un combat. L'incompétence et l'improductivité de ces hommes pour qui la réussite professionnelle était un support identitaire fondamental, les laissent anxieux de ne pouvoir occuper une position sociale valorisante et ni soutenir financièrement leur couple ou leur foyer. De la même manière, les personnages de Mes chers amis, Un éléphant, ça trompe énormément et Nous irons tous au paradis se caractérisent par leur incompétence qu'ils cumulent avec une absence totale d'intérêt pour leur famille ou leur épouse. Professionnels médiocres, époux infidèles et/ou tyranniques, pères démissionnaires, ils ne cessent de constater leur échec et semblent avoir totalement renoncé à être des figures d'autorité ou de stabilité, rompant ainsi avec le modèle patriarcal traditionnel.

\section{Des femmes castratrices}

S'il a des origines diverses, le manque de virilité de ces hommes les empêche de «se [distinguer] hiérarchiquement des femmes » et d'incarner cette masculinité hégémonique (Connell : 1992) dont la fonction est de «garantir la position dominante des hommes et la subordination des femmes » (Hirata, $2004: 71)$. Les femmes qui les entourent prennent alors l'ascendant sur eux et semblent parfois même chercher à se passer d'eux. Dans les deux cas, c'est la place des hommes au sein du couple, de la famille et/ou de la société qui est remise en cause.

Au début de La Grande Bouffe, le spectateur comprend que les quatre amis qui s'isolent dans un manoir pour le week-end, cherchent avant tout à fuir des femmes qui les étouffent. Ugo (Ugo Tognazzi), un restaurateur, est sous l'emprise d'une épouse sèche et autoritaire, tandis que Philippe (Philippe Noiret), un magistrat, nourrit une relation incestueuse avec sa nourrice, une femme opulente qui lui a donné le sein lorsqu'il était enfant et lui parle comme s'il en était encore un. Craignant qu'il n'ait des rapports sexuels avec une autre femme, elle se déclare prête à se «sacrifier» à 
nouveau, pour que cela «rest[e] en famille », à la suite de quoi Philippe, visiblement attiré par son décolleté, se plaint: «Nicooole! Tu me violes toujours!». Dans Vincent, François, Paul... et les autres, les femmes constituent une menace plus sourde mais néanmoins bien réelle. Vincent (Yves Montand), le chef d'entreprise quitté par sa femme Catherine (Stéphane Audran) en raison de ses infidélités, perd également sa maîtresse, la jeune Marie qui lui reproche son manque de prévenance. Affecté par ces ruptures, il se sent également menacé par son divorce. Il réunit alors ses amis pour leur demander de lui écrire des lettres de recommandation pour le juge, affirmant : «non, là, je suis obligé de me défendre, parce que sinon j'ai tous les torts contre moi »; et lorsque François lui demande contre quoi il cherche à se défendre, sa réponse trahit sa peur irrationnelle des femmes : « j'en sais rien justement, je sais pas ce qui peut arriver, tu sais, une femme, quand c'est plus ta femme, c'est...». Dans Mes chers amis, les épouses semblent être une menace permanente pour l'intégrité du groupe. Pleurnicheuses ou ensorceleuses, elles n'ont de cesse d'essayer d'empêcher les «virées tziganes» de leurs époux, longues escapades au cours desquelles ils roulent vers l'inconnu, jouant les pires farces à ceux qu'ils croisent sur leur chemin et retombant en enfance. Ainsi, Melandri, qui a enfin réussi à séduire une infirmière pour laquelle il avait eu un coup de foudre et qui l'a installée chez lui avec ses enfants, ne parvient plus à quitter le foyer, et finit, malheureux et dominé, par appeler ses amis à l'aide. L'ex-mari de l'infirmière, un médecin, participe à l'opération de «sauvetage » et il est catégorique: «diagnostic: cette femme constitue un danger pour toi. Traitement: la jeter ». Dans ce film comme dans les autres, tomber amoureux constitue une malédiction pour les hommes qui ne sortent jamais indemnes d'une relation avec une femme. Dans Nous irons tous au paradis..., les hommes, bien qu'ils se comportent globalement de manière odieuse envers leur famille, ne s'en présentent pas moins comme des victimes. Étienne (Jean Rochefort), le narrateur, persuadé que sa femme le trompe, se qualifie ainsi en voix-off de "pauvre garçon agressé en pleine jeunesse par la fureur des femmes ».

Dans Calmos!, Bertrand Blier exploite pleinement le motif de la femme castratrice et pousse l'humour misogyne à son comble en mettant en 
scène Paul (Jean-Pierre Marielle) et Albert (Jean Rochefort), un gynécologue et un proxénète, qui décident de «prendre le maquis » pour échapper à la menace que constituent non seulement leurs épouses mais également toutes les femmes. Ces deux hommes vivent dans l'angoisse permanente de la castration, symbolisée dès l'introduction du film par le vagin des patientes de Paul, comparé par le scénario et l'affiche du film à des vagina dentata qui menacent de l'engloutir (Rollet: 1999). Les deux amis fuient également les assauts répétés de leurs épouses qui les forcent à se livrer en permanence à des rapports sexuels qui les écœurent. Car dans Calmos!, les femmes se sont tellement libérées sexuellement qu'elles se comportent comme des furies, utilisant les hommes comme des objets sexuels pour combler leurs besoins, et faisant peser sur eux une terrible dictature de la performance qu'ils ne peuvent supporter. Les deux amis sont rejoints dans leur fuite par d'autres hommes qui cherchent à échapper à leurs épouses et aux hordes de femmes violeuses qui se sont constituées. Celles-ci, armées et vêtues de tenues militaires, ne tardent d'ailleurs pas à les poursuivre dans leurs chars d'assaut, pour les faire prisonniers et les forcer à s'accoupler à la chaîne avec elles.

Difficile donc de ne pas voir dans ce motif récurrent de la menace féminine une référence implicite ${ }^{10}$ au développement du mouvement féministe, comme le suggère le fait que ces films situent tous leur action dans une temporalité contemporaine et que l'offensive féminine semble avoir un caractère relativement récent, prenant les protagonistes par surprise.

\section{"C'est une opération de survie : elles ou nous "11 : la camaraderie comme rempart}

Cherchant à échapper aux femmes qui les entourent, les héros de ces films adoptent alors diverses stratégies, qui vont du choix d'un mode de vie marginal dans Les Valseuses au suicide collectif dans La Grande Bouffe, en passant par la prise du maquis dans Calmos! ou l'organisation de «virées tziganes" dans Mes chers amis. Mais quoi qu'il en soit, ces fuites symboliques ou réelles - se font entre hommes et permettent la reconquête

\footnotetext{
${ }^{10}$ Ou dans le cas de Calmos!, explicite.

${ }^{11}$ Citation du personnage de Paul dans Calmos!.
} 
d'une homosociabilité ${ }^{12}$ qui ne semble plus avoir sa place dans l'environnement désespérément mixte qui est le leur. L'amitié entre hommes est présentée comme une forme de sociabilité supérieure à toute autre, la seule qui ne soit pas décevante ou corruptrice. La camaraderie, fusionnelle et exclusive, apparaît alors comme le remède à tous les maux dont souffrent les hommes en crise. En dehors des protagonistes de Calmos!, qui se rencontrent dans la rue et «plaquent tout » pour fuir ensemble (parodiant ainsi un topos de la romance amoureuse), tous les autres se connaissent de longue date. Dans Mes chers amis, le narrateur Perozzi (Philippe Noiret) explique en voix-off : "nous voilà une fois de plus tous les quatre réunis. Y'en a même un cinquième, Sasarolli, qui va se joindre à nous. Mais ça, c'est un cas à part. Les vieux de la vieille, c'est nous. Camarades d'école ou de régiment, donc amis depuis toujours. Ce sont eux mes amis, mes chers amis ». De par leur caractère «immémorial », ces relations procurent donc un sentiment de stabilité sécurisant. L'amitié entre hommes survit là où le couple hétérosexuel et la famille échouent, et sa pérennité est d'autant plus précieuse que le monde semble plein de dangers.

On peut voir dans certains de ces films l'influence de la tradition américaine du «buddy movie » (littéralement «film de copains »), dans laquelle «deux hommes qui ont des personnalités et/ou sont issus de milieux très différents sont forcés de cohabiter », ce qui donne souvent lieu à des scènes comiques, jusqu'à ce que peu à peu, «leur incompréhension mutuelle se transforme en amitié et en respect»(Carroll, 2003: 74). Bertrand Blier est sans doute le réalisateur qui manie ces codes de la façon la plus explicite, surtout dans Les Valseuses où l'opposition entre Jean-Claude massif, viril, agressif - et Pierrot - plus fluet, moins confiant - est l'un des ressorts principaux de la comédie. Mais il n'est pas le premier à avoir importé le buddy movie en France et il s'inscrit au contraire dans une tradition initiée depuis le début des années 1950 et déjà identifiée par Noël Burch et Geneviève Sellier. Cette période qui se caractérise sur le plan cinématographique par une «remise en ordre patriarcale» suite aux

12 Daniel Welzer-Lang définit l'homosociabilité, comme «les relations sociales entre les personnes de même sexe, à savoir les relations entre les hommes ou les relations entre les femmes » (Welzer-Lang, 2000: 115). 
déstabilisations de l'Occupation, voit en effet se multiplier «les films qui reposent principalement sur une distribution masculine » et qui « relatent des histoires d'amitié virile et/ou de malfrats en bande » (Burch et Sellier, 2005 [1996] : 250). On peut citer par exemple Le Salaire de la peur (HenriGeorges Clouzot, 1953), Les Héros sont fatigués (Yves Ciampi, 1955), La Traversée de Paris (Claude Autant-Lara, 1956) ou bien les grands succès qui marquent le retour en force de Jean Gabin sur les écrans: Touchez pas au grisbi (Jacques Becker, 1954), Razzia sur la schnouf (Henri Decoin, 1954) et leurs épigones : Du rififi chez les hommes (Jules Dassin, 1955), Bob le Flambeur (Jean-Pierre Melville, 1956) et Rafles sur la ville (Pierre Chenal, 1958).

Burch et Sellier interprètent le retour de «ces histoires d'"hommes entre eux" qui exaltent la camaraderie » et "avaient quasiment disparu pendant l'Occupation (sauf dans les très rares films fascisants) » comme une indication « du besoin qui se fait sentir alors de réaffirmer la suprématie des valeurs masculines récemment malmenées » par la défaite de 1940 et l'Occupation (Burch et Sellier, 2005 [1996] : 250). Si les années 1970 sont traversées par des enjeux bien différents de ceux des années 1950, un parallèle peut néanmoins être établi. La présence récurrente de films valorisant la camaraderie en tête du box-office à partir de 1973 correspond en effet à un besoin de la part des réalisateurs - et des personnages qu’ils créent - de réaffirmer des valeurs viriles malmenées par le développement du mouvement féministe qui a dévalorisé et dénaturalisé les comportements machistes.

Mais tous les films de la période excluant les femmes pour mettre en scène l'amitié entre hommes ne peuvent pas être mis sur le même plan et les sept films étudiés appartiennent en fait à la frange la plus réactionnaire des « films d'hommes entre eux ». Parce que leurs personnages sont en crise et que les femmes sont présentées comme une menace, l'amitié masculine y prend une dimension éminemment défensive. Ils diffèrent sur ce point d'un bon nombre de comédies qui mettent en scène des hommes en bande durant la période, comme la série des Bidasses tournée par les Charlots, ou celle des Gendarme incarné par Louis de Funès. Les personnages de ces films ne sont absolument pas en crise et la réunion d'un grand nombre d'hommes à l'écran a avant tout une fonction comique qui témoigne tout au plus d'une 
volonté de relégation des femmes à des rôles subalternes - tendance de toute façen structurelle dans l'industrie du cinéma et plus encore dans la comédie. De la même façon, certains buddy movies des années 1970 se contentent de développer un comique de caractère et de situation par l'association de deux personnages que tout oppose, dans la lignée de La Grande Vadrouille (Gérard Oury, 1966) dont ils cherchent à reproduire le succès commercial ${ }^{13}$.

Les films étudiés dans cet article ne sont donc représentatifs que des orientations prises par une frange du champ cinématographique français qui est directement travaillée par l'évolution des rapports sociaux de sexe au cours des années 1970. On peut d'ailleurs repérer, à la même époque, une tendance similaire au sein du champ cinématographique hollywoodien. En effet, aux États-Unis, le genre du buddy movie qui a été initié dans les années 1930 par Laurel et Hardy n'a pendant longtemps été exploité que par des duos comiques (Abbott et Costello, Bing Crosby et Bob Hope, Dean Martin et Jerry Lewis). Mais on voit émerger dès le début des années 1970 un traitement plus sérieux et plus noir de l'amitié masculine, présentée comme une forme de résistance face au danger constitué par les femmes et un moyen d'échapper à l'ennui de la vie maritale et familiale, par exemple dans Butch Cassidy and the Sundance Kid (George Roy Hill, 1969), Easy Rider (Denis Hopper, 1969) ou Midnight Cowboy (John Schlesinger, 1969) (Carroll, 2003 : 74). Pour Molly Haskell et Robin Wood, ces films peuvent être lus sans équivoque comme une réaction de mâles blancs face aux évolutions des rapports de pouvoir aux États-Unis sous l'impulsion du mouvement féministe et du mouvement des droits civiques (Abbott, 2009 : 73).

Ainsi, en France comme aux États-Unis, l'industrie du cinéma produit de manière récurrente des univers fictifs exaltant une homosociabilité exclusive et excluante qui ne semble plus avoir sa place dans le monde réel. Unis face à l'adversité, leurs héros font corps, espérant parvenir à se protéger les uns les autres, dans un monde où les femmes semblent avoir déclaré la guerre aux hommes.

13 On pense par exemple à Le Cerveau (Gérard Oury, 1969), L'Emmerdeur (Édouard Molinaro, 1973), Fe suis timide mais je me soigne (Pierre Richard, 1978) ou La Chèvre (Francis Weber, 1981). 


\section{Homosociabilité et défense D’une MASGulinité hÉGÉMONIQUE MENAGÉE}

Mais au-delà de sa fonction défensive, l'homosociabilité remplit une fonction de «rééducation». En aménageant des espaces exclusivement masculins, les amis cherchent à faire prévaloir des valeurs viriles tombées en désuétude et à mener collectivement la reconquête d'une dignité perdue. Pour cela, ils mettent en place dans chaque cas un "programme » assez similaire : manger, boire, fumer et se distinguer. Cela permet non seulement le renforcement des liens d'amitié, mais encourage l'adoption par les membres du groupe d'un modèle de masculinité homogène, à même de leur assurer une position dominante dans la société. Dans un contexte où les personnages semblent menacés de féminisation, cette "police du genre $»^{14}$ s'exerce selon deux axes: par la promotion d'attitudes et de valeurs correspondant à un modèle de masculinité virile hégémonique et par le refoulement de tout attribut susceptible de questionner ce modèle ou de miner la « performance $»^{15}$ que constitue son adoption.

\section{Manger, boire et fumer}

Dans tous les films, les amis se livrent dès qu'ils sont réunis aux mêmes activités relevant de la régression orale : boire, manger et fumer. C'est $L a$ Grande Bouffe qui, comme son nom l'indique, accorde le plus d'importance à ce motif. La nourriture mais également l'alcool, omniprésents, sont source de jouissance puis de décadence, avant de se transformer en instrument de mort. De sa confection à sa dégustation, la gastronomie est donc élevée au rang d'art et grâce aux conseils d'Ugo, le cuisinier, les amis réalisent des recettes extrêmement complexes qui leur garantiront une mort à la fois extatique et spectaculaire. Dans Vincent, François, Paul... et les autres, les amis mangent régulièrement ensemble mais c'est surtout la consommation de l'alcool (souvent fort) et du cigare qui constitue la toile de fond de la

\footnotetext{
${ }^{14}$ L'expression « police du genre » est une traduction de l'anglais « gender policing ». Elle a été utilisée entre autres par Judith Butler pour qualifier l'ensemble des mécanismes d'assignation et de normalisation par lesquels une société donnée assure la pérennité des normes sexuées, en réprimant (physiquement ou psychologiquement, implicitement ou explicitement) les individus considérés comme « déviants » (Butler : 2005).

15 Depuis les années 1990, les Queer Studies ont développé le concept de «performativité du genre » pour insister sur le fait que le genre est avant tout une performance sociale apprise, répétée, et exécutée par les individus tout au long de leur vie (Butler : 2005).
} 
sociabilité masculine. Claude Sautet y développe une esthétique du «petit salon »- pièce sombre peuplée d'hommes en costume fumant des cigares et buvant du scotch - que l'on retrouve dans plusieurs de ses films (Max et les ferrailleurs, César et Rosalie, Mado). Cette obsession pour la nourriture, l'alcool et le cigare peut s'expliquer en partie par le fait que ces éléments sont perçus dans la « culture française » comme des vecteurs de convivialité et sont donc favorables au renforcement des liens de camaraderie si importants dans ces films.

Mais c'est dans Calmos! que la véritable fonction dévolue à la nourriture est le plus clairement explicitée. Les deux amis réfugiés à la campagne n'ont de cesse de manger et boire, activités perçues comme un « remède » et qui sont au cour de la «cure » de virilité qu'ils décident d'effectuer pour se défaire à jamais de l'influence des femmes. Dans ce cadre, le manque d'appétit est synonyme d'amollissement et s'empiffrer constitue un devoir. Ils règlent leur réveil pour se lever au milieu de la nuit et s'attablent autour d'un pâté en croûte en s'écriant : «au boulot!». Dans ce film comme dans les autres, les hommes mangent pour se prouver à euxmêmes et aux autres qu'ils sont des hommes. Les aliments consommés sont d'ailleurs sélectionnés dans cet esprit: viandes, charcuteries et autres gâteaux à la crème ne peuvent être digérés en grande quantité que par un estomac de «rude gaillard», comme l'affirment les personnages de La Grande Bouffe. Il s'agit donc de manger pour prouver son endurance et pour renouer avec un «instinct» carnivore et un appétit qui devraient caractériser, selon les différents groupes, les vrais hommes.

\section{Se distinguer}

La nourriture, les cigares et les alcools que consomment la plupart de ces hommes sont également des produits de luxe, utilisés de façon symbolique comme des outils de distinction de classe. En effet, dans les films étudiés, les hommes se réunissent en groupes socialement homogènes et appartiennent dans leur grande majorité aux classes aisées et dotées d'un capital scolaire et culturel important. Des intérêts de classe soudent cette appartenance commune et ils ne cessent de la réaffirmer collectivement comme pour défendre des privilèges menacés (Forbes, 1992 : 184). Dans La 
Grande Bouffe et Mes chers amis, les héros exercent des professions de « prestige » (juge, producteur de télévision, pilote de ligne, chef cuisinier dans une "grande maison ", architecte et chirurgien). Ils appartiennent donc à la grande bourgeoisie et si l'un d'eux n'a pas un sou (Lello dans Mes chers amis), ce n'est pas parce qu'il est issu d'un milieu populaire mais parce qu'il s'agit d'un noble désargenté qui n'a jamais travaillé. Vincent, François, Paul... et les autres situe plutôt son action dans la moyenne bourgeoisie. Les protagonistes sont patron d'une petite entreprise, médecin généraliste et écrivain, et si Jean, le plus jeune, n'est "qu'un ouvrier », cette infériorité sociale est symboliquement compensée par sa pratique de la boxe à un haut niveau et par le fait que son travail à l'usine n'est que temporaire (il décide à la fin du film de monter sa propre entreprise).

Ainsi, la connaissance des grands vins, le goût pour des meilleurs cigares et le culte des mets raffinés constituent pour ces hommes des moyens de mettre en avant leur culture. Lorsque Vincent (Vincent, François, Paul... et les autres) sert du vin à ses amis, il précise : «c'est un Don Pérignon ». Ugo (La Grande Bouffe) rappelle à ses amis : "Messieurs, nous ne sommes pas ici pour faire une orgie crapuleuse. » Paul (Calmos !) prône le fait de ne manger « que du naturel » et toujours « ce qu'il y a de meilleur ». En étalant leurs connaissances du patrimoine gastronomique français et international, ils expriment en permanence leur appartenance à une élite sociale, culturelle mais aussi sexuée. Car par opposition, les femmes (à l'exception d'Andréa dans La Grande Bouffe) sont incapables d'apprécier ces produits à leur juste valeur. Ainsi, dans Calmos!, alors qu'Albert supplie les femmes qui viennent de le capturer de lui donner quelque chose à manger, l'une d'elles lui tend un tube de lait concentré Nestlé - produit alimentaire bas de gamme et industriel par excellence - qui répugne profondément au captif.

À côté de cette culture gastronomique, les héros de ces films mettent régulièrement en avant une culture littéraire et artistique qui doit attester de leur goût pour les belles choses et de leur éducation. Ainsi les amis de $L a$ Grande Bouffe apprécient ensemble les peintures et photographies de nus que Philippe collectionne depuis son enfance et qu'il projette au mur pendant leur repas. Cette culture, si elle leur permet de se distinguer implicitement des prolétaires ignorants, est principalement utilisée contre les femmes qui 
sont non seulement idiotes et superficielles mais majoritairement incultes. Dans Calmos !, Paul déplore par exemple ne pas avoir pu parfaire sa culture depuis qu’il est marié : «j'ai jamais pu terminer Proust, parce que figure-toi qu'à chaque fois que j'essayais d'entamer le tome quatre, ça déclenchait un disque : 'chéri, on éteint'. »

Le cas des Valseuses est intéressant parce que Bertrand Blier prétend y mettre en scène des « marginaux ». Or, au cours de leur road-trip, les deux amis ne cessent d'affronter des personnes issues des classes moyennes ou populaires qu'ils cherchent constamment à humilier. Leur cavale commence par le vol de la voiture d'un patron de salon de coiffure, puis Jean-Claude défie un vigile de supermarché et ensuite, les deux amis volent les vélos de deux paysans, bloquent le tracteur d'un agriculteur et enfument avec leur pot d'échappement une famille qui pique-nique sur le bord de la route. Sous prétexte de s'attaquer à des symboles de la répression (un vigile) ou de l'ordre établi (les familles des classes moyennes), leurs attaques leur permettent de se dissocier de ceux qui sont qualifiés avec mépris à la fin du film de «travailleurs » et de «prolos ». Pourtant, les médias ont relayé le discours du réalisateur Bertrand Blier en qualifiant Pierrot et Jean-Claude de «loubards », « loulous », « zazous », « anarchistes » et « marginaux ». On peut au contraire considérer que ces deux hommes blancs et virils, aux prénoms bien français, qui ne cessent de boire des «petits rouges » et de manger du fromage, qui aiment la poésie et méprisent les prolétaires, incarnent justement à leur manière la France des dominants.

\section{Performances sexuelles et refoulement d'une ambiguïté homosexuelle latente}

Dans certains des films étudiés, à côté de manger, boire et se distinguer, les hommes ont, les uns devant les autres, des rapports sexuels avec les quelques femmes dont ils tolèrent la présence. C'est le cas dans $L a$ Grande Bouffe, où après le départ des prostituées, Andréa couchera avec les amis les uns après les autres, et dans Les Valseuses, où Marie-Ange (MiouMiou) aura des rapports sexuels avec les deux héros, en dépit du fait qu'elle n'y prend aucun plaisir. Dans ces films, outre la représentation systématique des femmes comme des objets sexuels toujours consentants, la possession physique des femmes est un acte public, occasion d'une démonstration de 
force visant à prouver sa virilité. Dans Les Valseuses, Jean-Claude se permet par exemple d'arrêter son ami Pierrot en plein acte pour lui «montrer l'exemple », se présentant comme « un ténor » en la matière. Pour ces deux amis, la sexualité est une compétition qui leur permet de se mesurer l'un à l'autre mais également de s'opposer ensemble à d'autres hommes. Tout au long du film, les femmes qu'ils agressent ou possèdent sexuellement ne sont que des objets médiateurs par le biais desquels ils cherchent à prendre l'avantage sur les hommes auxquels elles sont censées "appartenir». Ce rapport à la sexualité véhicule deux éléments caractéristiques d'une masculinité hégémonique: la compétitivité qui permet d'établir des hiérarchies au sein du groupe et entraîne les hommes à l'exercice de la domination, et l'objectification des femmes, qui permet de se définir en négatif par rapport à elles, comme différent et supérieur, et de faciliter l'exercice de la domination en les dépersonnalisant (Johnson, 1988).

Mais cette compétitivité qui encourage le développement d'une sexualité collective exhibitionniste, est alors porteuse d'un fort homoérotisme qui entretient une ambiguïté quant au potentiel désir homosexuel des membres du groupe. Dans Les Valseuses et La Grande Bouffe, les amis ont des rapports sexuels les uns à côté des autres avec les mêmes femmes et la mise en scène vise clairement à questionner la véritable source de leur désir qui semble parfois plus stimulé par la présence d'autres hommes que par celle d'une femme. Dans La Grande Bouffe, à mesure que le week-end avance, les protagonistes se mettent à dormir tous ensemble dans le lit de Marcello, les rapports sexuels prenant alors une dimension collective toujours à la limite du « dérapage ». Mais même lorsqu'aucun acte sexuel collectif n'est montré à l'écran, les liens d'homosociabilité unissant le groupe d'amis sont marqués par la recherche d'une solidarité indéfectible, et placés en conséquence sous le signe de la fusion. La volonté des protagonistes de ne former qu'un seul corps aboutit alors souvent à l'effacement des distances physiques et à un goût prononcé pour le contact charnel, toujours porteur d'une forte ambiguité homosexuelle. Par ailleurs, la misogynie du groupe et l'échec systématique du couple hétérosexuel semblent donner à l'homosexualité un caractère de fatalité, bien résumé dans Mes chers amis par le personnage de Melandri qui déclare après une soirée entre amis : «Ha ! 
Qu'est ce qu'on est bien entre hommes. Bon sang, quel dommage qu'on soit pas tous pédés!» Pourtant, si dans Les Valseuses Pierrot est - malgré luisodomisé par Jean-Claude qui lui explique qu'«entre copains, c'est normal », et si les comparses d'Un éléphant, ça trompe énormément découvrent que l'un d'entre eux préfère les hommes, l'homosexualité - perçue comme une faille dans les défenses viriles du groupe-demeure un tabou. Cette hypothèse, lorsqu'elle émerge, est alors immédiatement refoulée par les sarcasmes, le mépris ou le malaise.

Ces films confirment donc l'idée de R. W. Connell selon laquelle l'une des fonctions de l'homosociabilité est d'imposer une masculinité hégémonique, par la marginalisation ou la suppression des comportements qui - à l'instar de l'homosexualité - sont susceptibles de la remettre en question (Connell, 1987 et 1992). Par ailleurs, dans la mesure où ces hommes sont présentés comme « en crise », la police du genre exercée par le groupe afin d'assurer l'incorporation de la norme hégémonique par ses membres, est présentée comme un élément salvateur, à la fois nécessaire et positif, et la domination des hommes sur les femmes apparait alors comme un droit à reconquérir.

\section{ANTIFÉMINISME ET « GRISE DE LA MASGULINITÉ » : QUELLE RÉGEPTION GRITIQUE ?}

Ainsi, comme le soulignait Jill Forbes, un grand nombre de films français sortis en salle au cours des années 1970 sont porteurs d'un questionnement sur l'identité masculine -questionnement globalement absent des productions des années 1960 - qui semble traduire l'anxiété de leurs auteurs face à l'essor du mouvement féministe (Forbes, 1992: 183). Les figures d'« hommes en crise » dépossédés des prérogatives habituellement attachées à leur sexe, qui peuplent ces films, ne sont pas sans rappeler le «patriarcat défaillant » et les «pères châtrés » incarnés pendant l'Occupation par des acteurs comme Fernand Ledoux ou Raimu, ou bien les jeunes «hommes victimisés » de l'immédiat après-guerre, figures qui témoignent toutes de la « déstabilisation du patriarcat » suscitée par la défaite française de 1940 et l'occupation allemande (Burch et Sellier, 2005 [1996] : 89-97). Pourtant, nous avons vu que dans l'histoire du cinéma français, les aventures 
d'«hommes en bande » étaient plutôt caractéristiques du tournant des années 1950, moment marqué sur le plan cinématographique par une « remise en ordre patriarcale » se traduisant par la réaffirmation de valeurs viriles et la disparition ou la diabolisation des figures de femmes fortes du cinéma de l'Occupation (Burch et Sellier, 2005 [1996] : 245-278). Les productions étudiées dans cet article semblent ainsi associer tout à la fois les marques d'une déstabilisation masculine et celles d'une volonté plus ou moins agressive de retour en arrière (backlash) et de revanche contre les femmes. Ils concilient donc deux tendances en théorie opposées, à savoir la mise en scène d'hommes victimisés et l'affirmation de valeurs viriles. Cette spécificité leur confère a priori - bien qu'à différents degrés - une forte dimension antiféministe mais contribue également à brouiller leur réception.

On constate en effet que le qualificatif d'« antiféministe » n'est jamais avancé par la presse générale ou spécialisée de la période au sujet de ces films, et que les critiques formulées par les féministes à leur encontre se heurtent même au contraire à une grande circonspection. Le cas de Calmos ! est sur ce point symptomatique car ce film est le seul à mettre en scène des féministes et à assumer sans équivoque un traitement méprisant et misogyne de leur combat. Cette dimension est d'ailleurs si présente qu'elle est au cœur de la stratégie publicitaire déployée par Bertrand Blier et son équipe de production. Le réalisateur affirme à plusieurs reprises, lors d'entretiens, avoir voulu « concocter un canular pour célébrer à [sa] manière l'année de la femme » et les affiches du film utilisent des slogans tels que «le film qui remet les choses à leur place » ou «Soyez goujat, emmenez votre femme voir Calmos!» (Rollet, 1999 : 384). Pourtant, les journalistes s'obstinent à récuser les accusations de misogynie dont Calmos! fait l'objet :

Plusieurs critiques femmes se montrent d'une indulgence surprenante, soit en déclarant «Blier est misogyne... mais », soit en réfutant d'emblée une misogynie que le cinéaste revendique pourtant. Beaucoup de critiques préfèrent le considérer comme un «misanthrope » et en veulent pour preuve les mauvais traitements subis par les personnages masculins qui ne seraient guère mieux lotis que les personnages féminins. (Rollet, 1999 : 380) 
Cette mauvaise foi prend d'ailleurs parfois des proportions déroutantes, comme lorsqu'un journaliste fait de Bertrand Blier un admirateur des femmes en affirmant : « Mais cette frénésie de tout railler, de ne jamais laisser percer un bon sentiment, n'est-elle pas une pudeur? Pour faire ce film, il fallait une grande tendresse pour les femmes. Finalement, sous la farce, c'est d'amour qu'il s'agit ${ }^{16}$. » Pourtant, comme le souligne Brigitte Rollet, le fait que les féministes soient systématiquement représentées de façon humiliante - elles sont vulgaires, brutales et déshumanisées - et que leurs revendications soient discréditées - ce sont de véritables obsédées sexuelles qui violent les hommes sous prétexte de « libération » et dont les mots d'ordre se limitent à «Coït pour toutes » et «Chauds les clitos »- permet de conclure sans équivoque à l'antiféminisme et à la misogynie de Calmos! (Rollet, 1999: 384). Les Valseuses et Mes chers amis proposent un traitement similaire - bien que moins excessif- des personnages féminins, désincarnés et stéréotypés ou franchement antipathiques, si bien qu'ils n'offrent dans tous les cas aucune prise à l'identification des spectateurs/trices.

En revanche, les autres films étudiés ici ne donnent pas directement à voir le mouvement féministe, et le traitement qu'ils font des personnages féminins est globalement plus subtil et nuancé. Vincent, François, Paul... et les autres, Un éléphant, ça trompe énormément et Nous irons tous au paradis proposent même quelques personnages secondaires assez positifs d'épouses trompées et humiliées par leur mari, dont la révolte est présentée comme légitime et salvatrice. Gela a conduit de nombreux journalistes à prêter à ces films une dimension critique, affirmant qu'il s'agissait avant tout pour leurs réalisateurs de se moquer de la veulerie et de la misogynie de leurs personnages masculins. Cette lecture est d'ailleurs encouragée par Yves Robert lui-même qui affirme à propos d'Un éléphant, ça trompe énormément : « il y a longtemps que je voulais écrire un 'film d'hommes' qui ne soit pas à leur gloire. Le but était de faire rire de leurs mensonges, de leurs lâchetés, de leur vanité et de leurs faiblesses, et même de cette amitié dans laquelle ils trouvent parfois salut ou réconfort ${ }^{17}$. » Pourtant, quelles que soient les

${ }^{16}$ L’Express, 9 février 1976.

17 Yves Robert dans Le Figaro du 20 septembre 1976. 
intentions - réelles ou supposées - de leurs auteurs, force est de constater que la majorité de ces films ont suscité la sympathie et même l'empathie pour les personnages masculins et la prise au sérieux de leurs souffrances. Ce phénomène peut s'expliquer entre autres par l'utilisation d'acteurs appréciés du public et souvent associés à l'univers de la comédie (Jean Rochefort, Jean-Pierre Marielle, Philippe Noiret), qui créent d'emblée une complicité avec le public, ce qui rend difficile toute prise de recul des spectateurs par rapport au point de vue masculin (Forbes, 1992 : 184). Quoi qu'il en soit, la crise des personnages est considérée par beaucoup de contemporains comme symptomatique de l'«air du temps» et représentative de l'état d'esprit des hommes des années $1970^{18}$.

Vincent, François, Paul... et les autres, dont le style est empreint d'un plus grand réalisme, est en toute logique le film qui a suscité l'identification et l'adhésion la plus unanime de la part des journalistes. Un journaliste de $L a$ Croix qui intitule son article «VRAI!» affirme dans sa critique dithyrambique que ce film est «vrai, merveilleux, banal ${ }^{19} »$. Les Échos affirme qu'il s'agit d'« un film-miroir. L'image exacte, plus amère que douce, de notre vie, de la vôtre ${ }^{20}$ » et L'Humanité que « Sautet met le doigt sur certaines plaies du temps présent, en France et en 1974 ... » et que « tout cela constitue une œuvre singulièrement révélatrice de 'l'air du temps'21 ». L'Express va jusqu'à déclarer : «Si le film de Sautet nous bouleverse tant, c'est que nous sommes tous des Vincent, des François et des $\mathrm{Paul}^{22}$ » et $L e$ Nouvel Observateur: "Nous reconnaissons ce que César, Rosalie, Vincent, François, Paul, Jean et les autres sentent, souffrent, disent, font. Nous reconnaissons ce qui leur arrive. Ce sont les choses de la vie. De notre vie ${ }^{23}$.» Un éléphant, ça trompe énormément, Mes chers amis et Nous irons tous au

\footnotetext{
18 À défaut de posséder des sources permettant d'évaluer la nature de la réception de ces films par le grand public, j’analyse ici leur réception par les journalistes. Il est évident que les positions répertoriées ci-après ne sauraient être jugées représentatives de l'« opinion générale » du public, cependant elles ont pu influencer en partie la réception des films par les lecteurs de la presse écrite, à une époque où celle-ci demeure pour les Français le principal moyen d'information sur l'actualité cinématographique. Pour étudier cette réception journalistique, j'ai utilisé la base de données de la Bibliothèque du Film, qui répertorie tous les articles parus dans la presse nationale, régionale et spécialisée.

${ }^{19}$ La Croix, 7 octobre 1974.

${ }^{20}$ Les Échos, 3 octobre 1974.

${ }^{21}$ L'Humanité, 6 octobre 1974.

${ }^{22}$ L'Express, 6 octobre 1974.

${ }^{23}$ Le Nouvel Observateur, 28 octobre 1974.
} 
paradis suscitent également des commentaires similaires, même si les journalistes semblent moins unanimement enthousiasmés. Ils considèrent que ces film traitent d'un «sujet fort actuel ${ }^{24}$ », en donnant à voir «la souffrance aiguë ${ }^{25}$ » des personnages. La Grande Bouffe est finalement le seul film à déboucher sur une réception réellement contrastée, voire globalement négative. La dimension excessive et provocatrice du film ainsi que son dénouement, semblent avoir conduit les spectateurs à mettre à distance les quatre héros dont le complexe d'autodestruction n'émeut personne et suscite dans certains cas un rejet viscéral. Le film est qualifié de vulgaire, indécent et même misogyne, et l'opinion générale semble s'accorder à dire que pour que le suicide collectif des personnages émeuve, « [e]ncore faudrait-il que ce désespoir d'un pilote de ligne, d'un magistrat, d'un animateur de radio et d'un restaurateur soit le moins du monde motivé26 ». Malgré tout, face aux différents films, c'est la complaisance qui domine chez les critiques et les journalistes, et ces personnages d'hommes en crise sont pris très au sérieux ${ }^{27}$.

Ainsi, s'il est parfois difficile de savoir si les auteurs de ces films étaient guidés par une intention antiféministe, l'existence ou non de cette intention importe finalement peu. Car ces films ont bel et bien participé à la diffusion du thème de la « crise de la masculinité » qui triomphera à partir de la fin des années 1970 dans le cadre du backlash antiféministe, et dont le principal argument est que «le féminisme est allé trop loin» et que les hommes, désormais «castrés » sont en état de souffrance ${ }^{28}$. Ce discours, complètement déconnecté de la réalité juridique, économique et sociale de

24 Politique hebdo, 4 octobre 1976.

${ }^{25}$ Le quotidien de Paris, 18 août 1976.

${ }^{26}$ L'Aurore, 22 mai 1973.

${ }^{27}$ Je n'évoque pas ici la réception journalistique des Valseuses et de Calmos !, car la dimension provocatrice et volontairement politiquement incorrecte de ces films a suscité tant de débats au sein de la presse que la question de la crise personnelle traversée par les personnages masculin et de son aspect réaliste ou non est complètement mise de côté et n’est jamais évoquée.

${ }^{28}$ Le terme «backlash» (souvent traduit en français par l'expression « retour de bâton ») a été introduit en 1991 par la journaliste américaine Susan Faludi pour qualifier l'ensemble des discours et des pratiques mis en place par un groupe dominant dans le but de contester les droits acquis par un groupe dominé. En ce qui concerne les moments de backlash antiféministe, l'hypothèse de Susan Faludi est qu'ils surviennent systématiquement par vague, dans des contextes d'avancée des droits des femmes, c'est-à-dire à des moments où les femmes pourraient être en passe d'accéder à plus d'égalité vis-à-vis des hommes (Faludi : 1993). 
la France des années 1970, a émergé très rapidement après le début du mouvement féministe mais ne s'est imposé dans la sphère médiatique qu'autour de l'année 1979. Il s'agit d'une stratégie discursive qui vise non seulement à décrédibiliser le combat féministe mais également à mobiliser en faveur d'une reconquête des privilèges masculins perdus. Francis DupuisDéri souligne sur ce point qu'« un discours de crise peut avoir des effets sociopolitiques très concrets », et que « les élites et les classes dominantes ont souvent recours à un discours de crise pour encourager et légitimer la mobilisation des ressources à leur avantage », ainsi que pour discréditer des forces contestataires présentées comme la cause de la crise, et donc comme une menace (Dupuis-Déri, 2012 : 93). Les auteurs des films étudiés se sont donc inscrits - consciemment ou non- dans une tendance générale consistant à présenter les hommes comme les victimes collatérales de l'émancipation féminine et à s'émouvoir de leur sort. L'étude des émissions télévisées de débat et d'actualité des années 1970 témoigne également de ce déplacement des préoccupations des journalistes depuis la thématique de la «condition féminine » dont il est régulièrement question entre 1970 à $1978^{29}$, vers la thématique de la «condition masculine » qui prend le dessus à partir de 1979. Une série d'émissions qui se veulent "post-féministes », intitulées par exemple «Le féminisme a-t-il changé les hommes ${ }^{30}$ ? » ou « Ça va les hommes ${ }^{31}$ ? », proposent alors soit de tirer le bilan de l'évolution des hommes sous l'influence du féminisme, soit, dans un registre plus alarmiste, de critiquer les effets du féminisme sur les hommes.

L'émission Apostrophes du 9 janvier 1981 est particulièrement représentative de cette tendance. Intitulée «Et si on parlait des hommes? » elle est introduite par son présentateur Bernard Pivot par la déclaration suivante: «En six ans d'Apostrophes, j’ai bien dû vous proposer une demidouzaine d'émissions sur les femmes en général et sur le féminisme en

\footnotetext{
29 La consultation des archives de l'Institut national de l'audiovisuel montre que de nombreuses émissions d'actualité et de débat portent entre 1976 et 1978 sur le féminisme et la place des femmes dans la société. Leurs titres ("Femmes en révolte », «La condition féminine de George Sand à Gisèle Halimi », « De la femme aux femmes », « La révolte des femmes ", "La sexualité racontée par les femmes», ou "Itinéraires de femmes", «Femmes, femmes, femmes») témoignent d'une volonté de prendre au sérieux cette question et de se placer du point de vue des femmes.

${ }^{30}$ Apostrophes du 22 février 1980.

31 Émission de débat diffusée le 3 octobre 1980.
} 
particulier. Mais sur les hommes, rien!», à la suite de quoi il propose de « rétablir l'égalité ». Or, Bertrand Blier est ce soir-là l'un des invités d'honneur de l'émission. Il y revendique le fait de n'avoir pas cédé au cours des dernières années à ce qu'il qualifie de «mode », et d'avoir persisté à «parler des hommes». Ses films sont alors présentés - avec une grande bienveillance - par Bernard Pivot comme les prémisses d'une « riposte » masculine au féminisme et comme un avertissement quant à l'émergence d'une "crise de la masculinité », considérée par la majorité des invités de l'émission comme une réalité sociologique.

Il semble finalement que si seuls quelques réalisateurs isolés revendiquent ouvertement - à la manière de Bertrand Blier - vouloir mener une « riposte » et une « contre-attaque », nombreux sont ceux qui ont contribué à cette offensive antiféministe en participant à la diffusion de ce nouveau stéréotype du cinéma français des années 1970 qu'est l'« homme en crise ».

\section{BIBLIOGRAPHIE}

Aввотт Stacey (2009), Angel, Detroit, Wayne State University Press.

Burch Noël et Geneviève SELLIER (2005 [1996]), La Drôle de guerre des sexes du cinéma français 1930-1956, Paris, Armand Colin.

BUTLER Judith (2005), Trouble dans le genre: le féminisme et la subversion de l'identité, Paris, La découverte [trad. de Gender Trouble: Feminism and the Subversion of Identity, New-York, Routledge, 1990 par Cynthia Kraus].

Carroll Bret E. (2003), American Masculinities : A Historical Encyclopedia, Sage Publications.

Connell R. W. (1987), Gender and Power : Society, the Person, and Sexual Politics, Stanford, Stanford University Press.

Connell R. W. (1992), Masculinities, Cambridge/Sidney/Berkeley, Polity Press/Allen \& Unwin/University of California Press.

Dupuis-Déri Francis (2012), «Le discours de la 'crise de la masculinité' comme refus de l'égalité entre les sexes: histoire d'une rhétorique antiféministe », Recherches féministes, vol. 25, n 1, 2012, p. 89-109.

FALudi Susan (1993), Backlash: la guerre froide contre les femmes, Paris, Des femmes [trad. de, (1991) Backlash: The Undeclared War Against American Women, New York, Crown, par Lise Éliane Pomier, Élise Chatelain, Thérèse Réveillé].

Ferro Marc (1973), «Le film, une contre-analyse de la société ? », Annales. Économies, Sociétés, Civilisations, n 1, 28e année, p. 109-124.

FORBES Jill (1992), The Cinema in France after the New Wave, Londres, BFI. 
Hirata Helena, Françoise Laborie, Hélène Le DoAre, Danièle Sentier (2004), Dictionnaire critique du féminisme, 2e édition augmentée, Paris, PUF.

Johnson Miriam (1988), Strong Mothers, Weak Wives, Berkeley, University of California Press.

Mulvey Laura (1975), "Visual Pleasure and Narrative Cinema », Screen, XVI, n 3, p. 6-18.

ROLLET Brigitte (1999), "Un canular anti-MLF : Calmos! de Bertrand Blier », dans Christine BARD (dir.), Un siècle d'antiféminisme, Paris, Fayard, p. 379-386.

Simsi Simon (2000), Ciné-Passions. 7e art et industrie de 1945 à 2000, Paris, Dixit.

Welzer-Lang Daniel (2000), «Pour une approche non-féministe non homophobe des hommes et du masculin », dans Daniel WELzERLANG (dir.), Nouvelles approches des hommes et du masculin, Toulouse, PU Mirail. 